\title{
Quantitatively Analyzing the Influence of Online News Acquisition on Public Web Searching Behaviors
}

\author{
Shueh-Cheng $\mathrm{Hu}^{1}$ and I-Ching Chen ${ }^{2+}$ \\ ${ }^{1}$ Department of Computer Science \& Comm. Engineering Providence University Taichung 43301, Taiwan, \\ ROC \\ ${ }^{2}$ Department of Information Management Chung Chou University of Science and Technology Chang Hua \\ 51003, Taiwan, ROC
}

\begin{abstract}
Administrators of public affairs usually ask "What are the problems concerning people mostly?" When people widely use online search services to find out information of interest and required knowledge, the search volume index (SVI) can serve as the instrument for nowcasting some phenomenon and problems that concern public, such as spread of epidemic diseases, economic trends, etc. However, if the search volume index is significantly influenced by media coverage, its precision of gauging problems will be questioned. That's because people who were influenced by media reporting, tend to conduct search due to curiosity rather than self-awareness. With the effect of media coverage on public searching behavior, the SVI cannot be used alone to nowcast or forecast things. The present work aims to investigate the influence of online news acquisition on the SVI. With attenuation of media influence, administrators of public affairs can gain insight into note-worthy search trends and the problems bearing public awareness, which facilitate decision making about the corresponding solutions.
\end{abstract}

Keywords: Public problems, search volume index, media coverage, regression analysis

\section{Introduction}

Traditionally, public affairs administrators try to understand issues concerning most citizens by using questionnaires, phone interviews, etc. In the age of Internet, because many people rely on search engines to find out information regarding their interests and concerns, correspondingly, issues concerning public could be to certain extent reflected by collective searching behavior. People's collective searching behavior could be measured by the so called search volume index (SVI). In fact, several prior works reported that SVI had been used to successfully forecast the spreading of diseases [1,2], trend of financial market [3, 4], and sales of products and services [5]. The fundamental assumption of the SVI being able to precisely forecast a particular issue is that most people conduct Web search due to their self-awareness or self-interest regarding the issue, rather than pure curiosity. As Ginsberg et.al stated in their work detecting influenza epidemics via Web query data [6], they assumed that people conducting Web search with specific keywords have motivation to avoid or cure diseases.

However, people conduct Web search because of not only their self-awareness and interests, but also curiosity, thirst for knowledge, and other factors. Among many factors, media coverage is the most significant one influencing peoples' Web search behaviors [7-9]. That means many people start Web searching only after receiving relevant messages from media, and in this case, the SVI obviously cannot serve as an effective instrument to nowcast and forecast issues that are closely related to public interests and concerns.

\footnotetext{
+ Corresponding author. Tel.: +886-936-831-021

E-mail address: shuehcheng@gmail.com; jineandya@gmail.com
} 
In light of media coverage's influence on search volume index that indicates emerging public issues and gauges their severities, the present work aims to quantitatively analyze the influence of online news acquisition on the public Web searching behaviors. With the findings, SVI's capability of nowcasting or forecasting public issues could be re-calibrated based on the extent to which media coverage influence SVI.

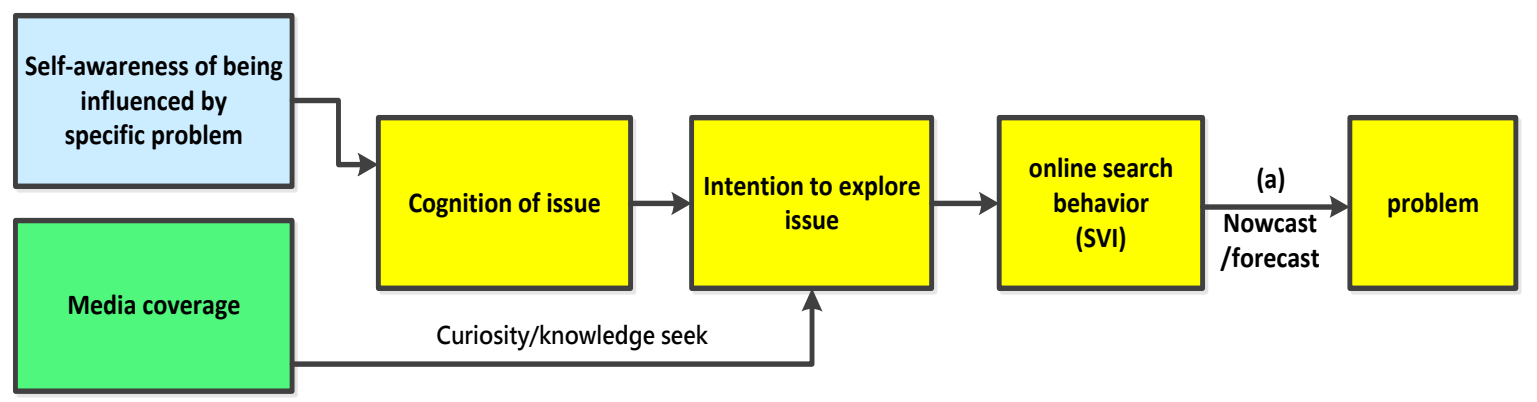

Fig. 1: Factors influencing public Web search

\section{Prior Works Review}

The search volume index is a normalized query share (relative) index, rather than a counter (absolute) index [5]. The SVI of one particular set of keywords reflects the percentage deviation of the searching behavior, comparing with the share of the same query on Jan 2004. Many phenomena and problems could be revealed through observing and correctly interpreting a series of SVI data. For example, the line graph in figure 2 shows the series of SVI associated with the query "PM2.5" in Taiwan during the last 5 years. The index soared 3 times, which means the importance of air pollution issues did repeatedly gain widely awareness and attention while increasing number of respiratory diseases and death was observed.

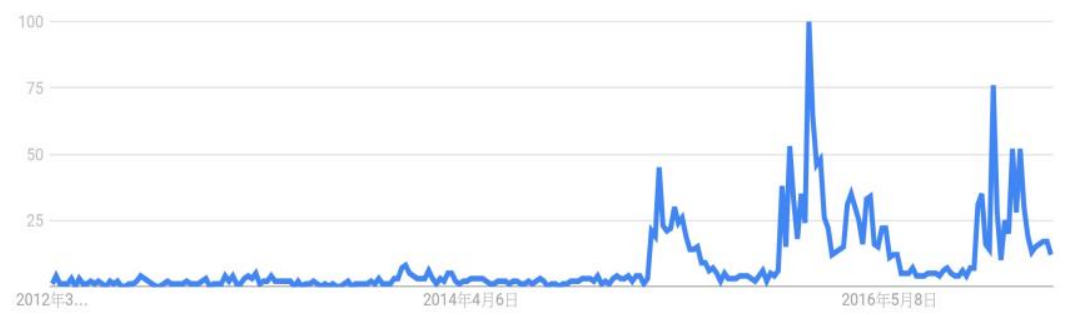

Fig. 2: SVI of query "PM2.5"

Comparing with other traditional methods to understand and measure public opinions or concerns, people quickly found that SVI is a good option for nowcasting and forecasting social phenomena and public issues, because of its immediacy, easy availability, standardized numerical format (efficient processing), etc. Herman Anthony Carneiro and Eleftherios Mylonakis built a system for monitoring disease outbreaks based on the Google trends service, and they reported that their system can raise alarms 7 to 10 days earlier than the conventional disease alarming system [10]. Besides public health issues, researchers also found that the SVI also serve as a good indicator for a company's stock price and trade volume while the economy condition is stable, especially for technology companies [11].

Brian Weeks and Brian Southwell study the relationships between media coverage and public Web searching behavior, they pointed that the media coverage volume on a particular issue can precisely predict the SVI associated with the same issue, although the media influence is transient [7]. Matthew Ragas, et al. also found that the volume of media coverage on an issue has significant impact on people's Web searching behavior [8]. Given these facts, the SVI must be adjusted based on the influence brought by media coverage, before it is applied to nowcast or forecast things.

\section{Research Method}

The present work tried to investigate the correlation between public news acquisition and their Web searching behaviors, in 3 different domains including social phenomenon, commercial product, and public policy. And then compare and contrast the correlations in different domains. 


\subsection{Data collection}

For each subject domain, a number of representative keywords were selected. Then, the data reflecting changes in public news acquisition and Web search were collected from the Google trends service (trends.google.com). Based on the Google's dominance on global search service, the data from Google should possess enough validity for our purpose. Taking a longitudinal viewpoint into account, the data during the last 5 years were retrieved; one pair of data items was collected per week.

\subsection{Regression analysis}

To investigate the relationship between the public news acquisition behavior (the explanatory variable: PM25_News) and the Web search behavior (the response variable: PM25_Web), simple regression analysis and the $\mathrm{R}$ language were applied in this work.

Taking the query "PM2.5" as an example, 3 different regression (linear, polynomial, power) models were tried, based on the observation of regression line and the scatter plot of subject data, linear model is a better option for describing the relationship between the explanatory and response variables, as shown in figure 3 .

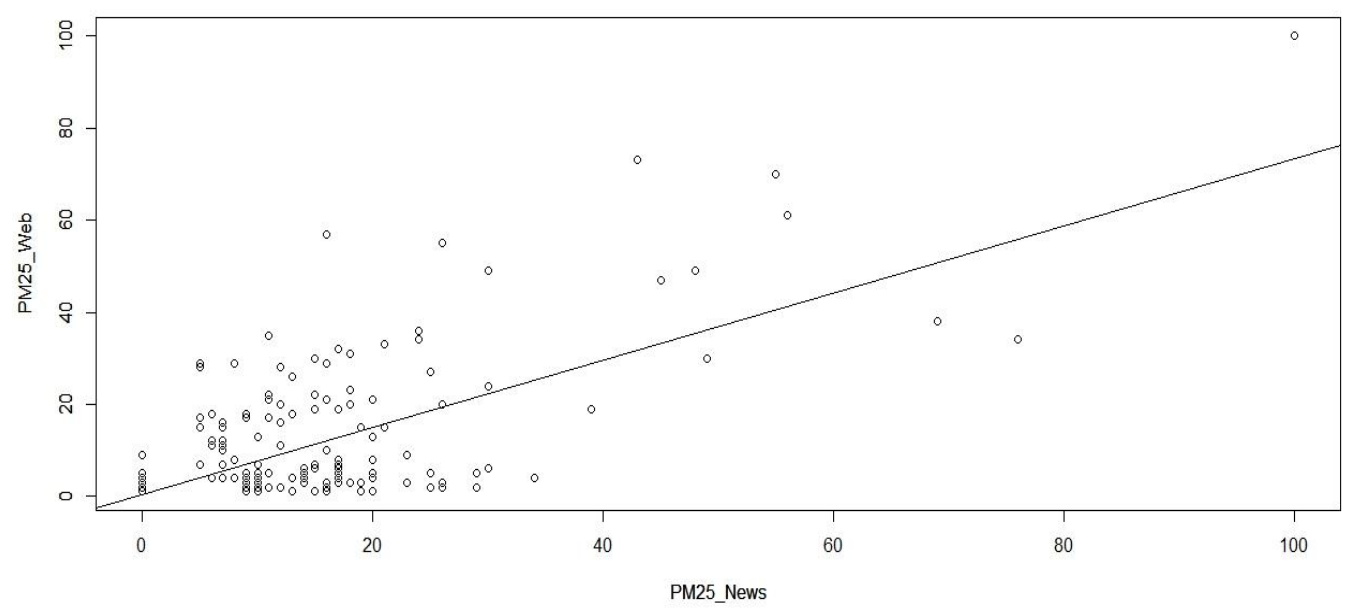

Fig. 3: Linear regression model of news acquisition and Web search on "PM2.5"

\section{Analysis and Discussion}

The investigation of regression analysis results determines the overall quality of the regression model describing the relationship between the public news acquisition and their Web search behaviors, which further can guide the adjustment of SVI's effectiveness on nowcasting or forecasting public issues.

\subsection{The query of "PM2.5"}

Keep taking the query of "PM2.5" as the example, the F-test $(2.2 \mathrm{e}-16<0.001)$ for overall significance in the analysis of variance (ANOVA) indicates that we can reject the null-hypothesis and conclude that the regression model provides a better fit than the intercept-only model. As table 1 shows, the predictor variable of PM25_News is significant because of its p-values is close to zero $(<0.001)$, and the corresponding coefficient (0.73070) represents that the mean change in the response variable (PM25_Web) for one unit of change in the predictor variable while holding other predictors in the model constant.

Table I: Summary of Regression (Pm25_Web Pm25_News))

Residuals:

\begin{tabular}{lllll}
\hline Min & $1 \mathrm{Q}$ & Median & $3 \mathrm{Q}$ & Max \\
\hline-21.845 & -5.889 & 0.688 & 2.688 & 44.997 \\
\hline Coefficients: & Estimate & Std. Error & t value & p-value \\
\hline
\end{tabular}




\begin{tabular}{lllll}
\hline (Intercept) & 0.31234 & 0.81074 & 0.385 & 0.7 \\
\hline PM25_News & 0.73070 & 0.04781 & 0.04781 & $<2 \mathrm{e}-16 * * *$ \\
\hline
\end{tabular}

Residual standard error: 9.823 on 259 degrees of freedom

Multiple R-squared: 0.4742 ， Adjusted R-squared: 0.4722

F-statistic: 233.6 on 1 and 259 DF, p-value: $<2.2 \mathrm{e}-16$

To further check the fitness of the model, the plot in figure 4 indicates that the residuals tend to close to the zero line throughout the range of fitted values. In other words, the model is correct on average for all fitted values. That appearance means the residuals was not either systematically high or low, i.e., there was randomness in the residuals.

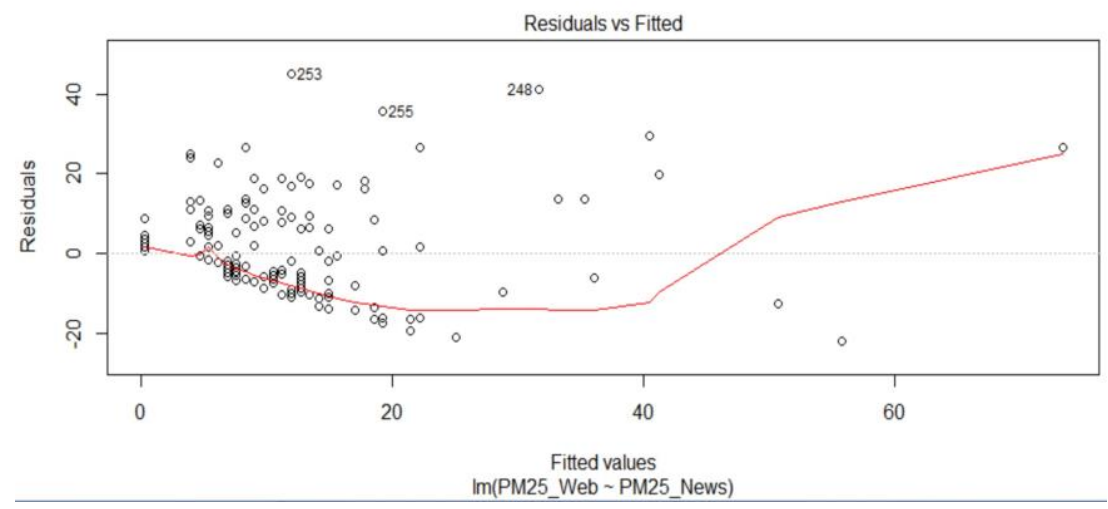

Fig. 4: Residual plot of the regression model on "PM2.5"

Linear regression using ordinary least squares identifies a formula that minimizes the distances (residuals) between the fitted line and all of the data items. R-squared, also known as the coefficient of determination, is an instrument for measuring how close the data points are to the fitted regression line, it is the percentage of the response variable variation that could be explained by the regression model. The adjusted $\mathrm{R}$ square $(0.4722)$ in the "PM2.5" case shows an adequate value in predicting human behaviors or social phenomenon, which usually are not predictable as science phenomenon.

\subsection{The query of "Cancer"}

To compare and contrast cases in different subject domain, the query of "cancer" was also taken into account since that illness has being topped the death cause rank in Taiwan for many years, so it should gain wide awareness and attention even media does not cover it intensively during the last years. With the similar procedure for data collection and analysis, we obtained a regression model describing the relationships between public new acquisition and Web searching on "cancer" as shown in figure 5, and its summary is listed in table 2.

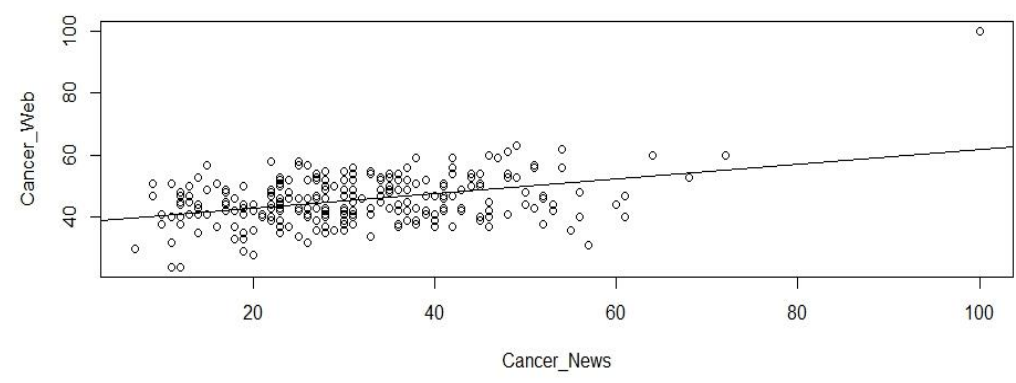

Fig. 5: Linear regression model of news acquisition and Web search on "Cancer"

Table II: Summary of Regression (Cancer_Web Cancer_News))

Residuals: 


\begin{tabular}{lllll}
\hline Min & $1 \mathrm{Q}$ & Median & $3 \mathrm{Q}$ & Max \\
\hline-20.552 & -5.261 & -0.296 & 5.281 & 38.296 \\
\hline Coefficients: & Estimate & Std. Error & t value & p-value \\
\hline (Intercept) & 38.09487 & 1.14384 & 33.304 & $<2 \mathrm{e}-16^{* * * *}$ \\
\hline Cancer_News & 0.23609 & 0.03383 & 6.979 & $2.5 \mathrm{e}-11^{* * *}$ \\
\hline
\end{tabular}

Residual standard error: 7.255 on 258 degrees of freedom

Multiple R-squared: 0.1588， Adjusted R-squared: 0.1556

F-statistic: 48.71 on 1 and 258 DF, p-value: $<2.498 \mathrm{e}-11$

The data in table 2 shows that the predictor variable of Cancer_News (p-value $=2.5 \mathrm{e}-11$ ) is significantly related to the response variable of Cancer_Web. However, the slope $(0.23609)$ of the relationship is not that steep as the one in the query of "PM2.5", which means the change in the predictor variable contribute less to the change of the response variable in the case of "cancer". Comparing with the high slope in the case of "PM2.5", the SVI reflecting public Web search on "cancer" should has stronger capability of nowcasting things that attract people's self-awareness without too much influence by media coverage.

To check the fitness of the model, the plot in figure 6 indicates that the residuals was not either systematically high or low, i.e., there was randomness in the residuals. Along with other statistical indices, the randomness of residuals indicates that the regression model provides good fitness to the sampled data.

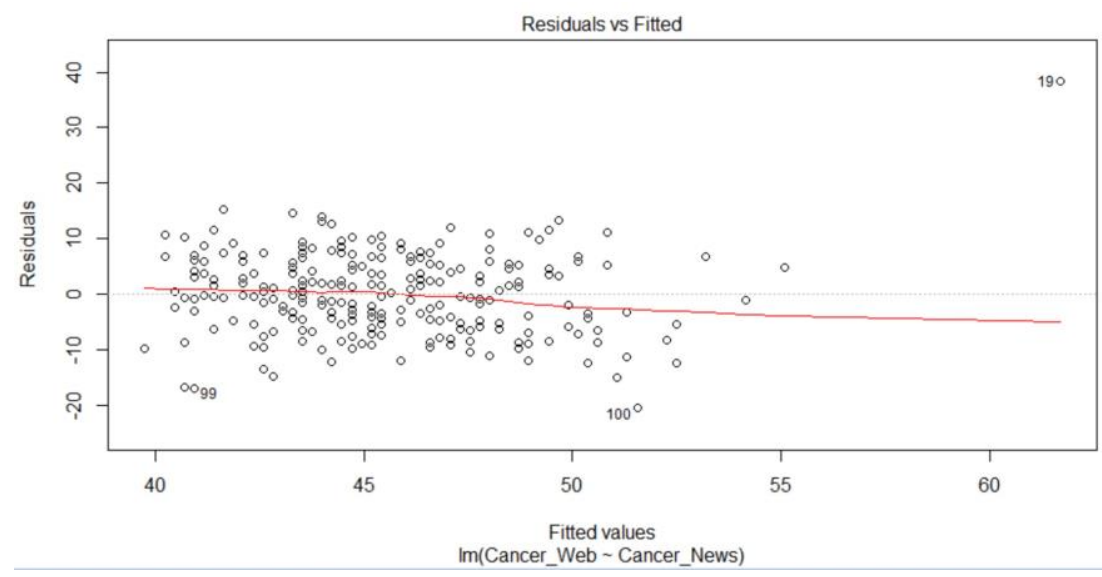

Fig. 6: Residual plot of the regression model on "Cancer"

\section{Conclusions}

\subsection{Impacts}

To respond public issues quickly and precisely, public affairs administrators tried to find efficient and effective ways to grasp issues that interest or concern the public. The search volume index that is available through search services, has been successfully used to nowcast and forecast some social phenomenon or human behaviors. However, because media coverage usually incurs people's curiosity, thirst for knowledge, and other factors leading to Web search, it is necessary to modulate the influence of public news acquisition on their Web search behavior that could be measured by the SVI.

To establish the basis for the modulation, the present work quantitatively investigated the relationship between public news acquisition and their Web search behaviors by using regression analysis techniques. The findings imply that anyone who plans to use the SVI as the indicator for nowcasting something must conduct solid analysis works and check the prediction model's quality before being able to correctly interpret the meaning of changes in the SVI on a query associated with a particular issue subject.

\subsection{Future directions}


The regression analysis results for queries in different subject domains vary, in other words, there is no common regression formula fitting all conditions. In consequence, public affairs administrators who want to identify and measure public issues by applying the SVI need to conduct regression analysis based on the particular queries of interest. Therefore, it is worthy to develop an automatic and adaptable procedure for conducting volume of regression works that are relevant to pubic issues.

Moreover, to find out whether there is any causal relationships between the media coverage and public Web search behavior, advanced statistical techniques such as time series analysis could be applied.

\section{Acknowledgment}

This research work has being funded by the grant from the Ministry of Science and Technology, Taiwan, ROC, under Grant No. MOST 105-2221-E-126-012-. We deeply appreciate their financial support and encouragement.

\section{References}

[1] S. Cook, C. Conrad, A. L. Fowlkes, and M. H. Mohebbi, "Assessing Google flu trends performance in the United States during the 2009 influenza virus A (H1N1) pandemic," PloS one, vol. 6, p. e23610, 2011.

[2] J. R. Ortiz, H. Zhou, D. K. Shay, K. M. Neuzil, A. L. Fowlkes, and C. H. Goss, "Monitoring influenza activity in the United States: a comparison of traditional surveillance systems with Google Flu Trends," PloS one, vol. 6, p. e18687, 2011.

[3] T. Preis, H. S. Moat, and H. E. Stanley, "Quantifying trading behavior in financial markets using Google Trends," Scientific reports, vol. 3, 2013.

[4] Y. Carrière-Swallow and F. Labbé, "Nowcasting with Google Trends in an emerging market," Journal of Forecasting, vol. 32, pp. 289-298, 2013.

[5] H. Choi and H. Varian, "Predicting the present with google trends," Economic Record, vol. 88, pp. 2-9, 2012.

[6] J. Ginsberg, M. H. Mohebbi, R. S. Patel, L. Brammer, M. S. Smolinski, and L. Brilliant, "Detecting influenza epidemics using search engine query data," Nature, vol. 457, pp. 1012-1014, 2009.

[7] B. Weeks and B. Southwell, "The symbiosis of news coverage and aggregate online search behavior: Obama, rumors, and presidential politics," Mass Communication and Society, vol. 13, pp. 341-360, 2010.

[8] M. W. Ragas, H. L. Tran, and J. A. Martin, "Media-induced or search-driven? A study of online agenda-setting effects during the BP oil disaster," Journalism Studies, vol. 15, pp. 48-63, 2014.

[9] M. W. Ragas and H. Tran, "Beyond Cognitions A Longitudinal Study of Online Search Salience and Media Coverage of the President," Journalism \& Mass Communication Quarterly, vol. 90, pp. 478-499, 2013.

[10] H. A. Carneiro and E. Mylonakis, "Google trends: a web-based tool for real-time surveillance of disease outbreaks," Clinical infectious diseases, vol. 49, pp. 1557-1564, 2009.

[11] X. Rui, "Google Search Volume Index: Predicting Returns, Volatility and Trading Volume of Tech Stocks," Economics, Duke, 2015. 